(1) CrossMark

Cite this: RSC Adv., 2016, 6, 61267

DOI: 10.1039/c6ra90050j

www.rsc.org/advances

\title{
Correction: IDO as a drug target for cancer immunotherapy: recent developments in IDO inhibitors discovery
}

\author{
Shan Qian, ${ }^{\star a}$ Man Zhang, ${ }^{a}$ Quanlong Chen, ${ }^{a}$ Yanying He, ${ }^{a}$ Wei Wang ${ }^{a}$ \\ and Zhouyu Wang*b
}

Correction for 'IDO as a drug target for cancer immunotherapy: recent developments in IDO inhibitors discovery' by Shan Qian et al., RSC Adv., 2016, 6, 7575-7581.

In Fig. 2 of the original manuscript, the structures given for Indoximod and Epacadostat were incorrect. In addition, the structure labelled "GDC-0919" was labelled incorrectly; the correct label is "GDC-0919 analogue". The corrected Fig. 2 is shown below.
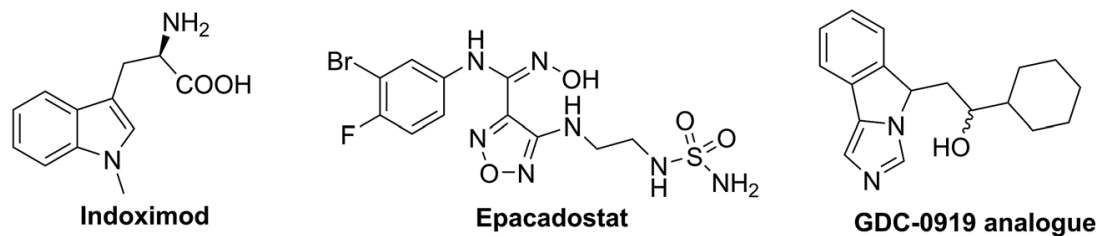

Fig. 2 Three small-molecule compounds in clinical trials.

In Section 3 of the manuscript, "Therapeutic strategies and challenges of IDO inhibitors in cancer immunotherapy", an incorrect $\mathrm{IC}_{50}$ value was given for Epacadostat, and a citation to ref. 17 of the original manuscript was omitted. The corrected text should read:

"Epacadostat (INCB024360) was obtained following a high throughput screening (HTS) of Incyte's corporate collection $\left(\mathrm{IC}_{50}=\right.$ $0.072 \mu \mathrm{M}){ }^{17,18,}$

In Section 4 of the manuscript, "Structure-based IDO inhibitors design", a citation to ref. 26 of the original manuscript was omitted. The corrected text should read:

"Based on the co-crystal structure of IDO with PIM, Roerhrig et al. ${ }^{26}$ and Huang et al. ${ }^{30}$ discovered independently that $1 H-1,2,3-$ triazole might be a new key pharmacophore of potent IDO inhibitors."

Additionally, the following discussion should be added to the end of Section 4, and the reference cited as ref. 1 of this correction should be added:

"As Roehrig et al. were concentrating on developing 4-aryl triazoles as potent IDO inhibitors, they used computational structurebased methods to design more triazole-based IDO inhibitors with low molecular weight and high efficiency. The most potent compound has an $\mathrm{IC}_{50}$ value in the nanomolar range both in the enzymatic and in the cellular assays. ${ }^{\prime \prime}$

The Royal Society of Chemistry apologises for these errors and any consequent inconvenience to authors and readers.

\section{References}

1 U. F. Roehrig, S. R. Majjigapu, A. Grosdidier, S. Bron, V. Stroobant, L. Pilotte, D. Colau, P. Vogel, B. J. Van den Eynde, V. Zoete and O. Michielin, J. Med. Chem., 2012, 55, 5270-5290. 